\title{
Two-Dimensional Nonlinear Propagation of Ion Acoustic Waves through KPB and KP Equations in Weakly Relativistic Plasmas
}

\author{
M. G. Hafez, ${ }^{1,2}$ M. R. Talukder, ${ }^{3}$ and M. Hossain Ali $^{2}$ \\ ${ }^{1}$ Department of Mathematics, Chittagong University of Engineering and Technology, Chittagong 4349, Bangladesh \\ ${ }^{2}$ Department of Applied Mathematics, University of Rajshahi, Rajshahi 6205, Bangladesh \\ ${ }^{3}$ Department of Applied Physics and Electronic Engineering, University of Rajshahi, Rajshahi 6205, Bangladesh
}

Correspondence should be addressed to M. G. Hafez; golam_hafez@yahoo.com

Received 28 April 2016; Accepted 25 July 2016

Academic Editor: Boris G. Konopelchenko

Copyright (C) 2016 M. G. Hafez et al. This is an open access article distributed under the Creative Commons Attribution License, which permits unrestricted use, distribution, and reproduction in any medium, provided the original work is properly cited.

\begin{abstract}
Two-dimensional three-component plasma system consisting of nonextensive electrons, positrons, and relativistic thermal ions is considered. The well-known Kadomtsev-Petviashvili-Burgers and Kadomtsev-Petviashvili equations are derived to study the basic characteristics of small but finite amplitude ion acoustic waves of the plasmas by using the reductive perturbation method. The influences of positron concentration, electron-positron and ion-electron temperature ratios, strength of electron and positrons nonextensivity, and relativistic streaming factor on the propagation of ion acoustic waves in the plasmas are investigated. It is revealed that the electrostatic compressive and rarefactive ion acoustic waves are obtained for superthermal electrons and positrons, but only compressive ion acoustic waves are found and the potential profiles become steeper in case of subthermal positrons and electrons.
\end{abstract}

\section{Introduction}

Rigorous theoretical and numerical studies on electronpositron-ion (epi) relativistic plasmas are conducted by several researchers [1-19] due to their potential applications and significance in understanding different types of collective processes in astrophysical as well as space [20-36] and laboratory [37-43] plasmas. The experimental observations are also carried out by many experimentalists [37-43] under different plasma conditions in order to uncover the physical processes involved. On the other hand, one-dimensional nonlinear dynamics of the epi relativistic plasmas are studied by a number of researchers [1-19]. Malik [14] has studied the effect of electron inertia on Kadomtsev-Petviashvili (KP) solitons in relativistic plasmas. Malik [15] has also studied the ion acoustic (IA) solitons in a weakly relativistic magnetized warm plasma. Singh et al. $[18,19]$ have investigated the effect of electron inertia on one-dimensional evolution of solitons and small amplitude of solitons in two-fluid weakly relativistic plasmas through usual Korteweg-de Vries (KdV) equation. Very recently, Hafez and Talukder [11] have studied the weakly relativistic influence on the IA solitary waves in onedimensional case by considering nonextensive electrons and isothermal positrons. Hafez et al. [12] have also investigated the one-dimensional weakly relativistic effects on the electrostatic IA positive as well as negative solitons composing nonextensive electrons and positrons. Only a few works [44, 45] are devoted to studying the two-dimensional nonlinear dynamics of ion acoustic (IA) waves in such plasmas. Han et al. [44] have investigated the existence of IA solitary waves and their interaction in two-dimensional thermal plasmas considering weakly relativistic hot ions and isothermal electrons by solving two KdV equations for small but finite amplitude solitary waves. They found that only compressive solitons for isothermal electrons and the phase shifts of the colliding solitary waves strongly depend on the colliding angle. Masood and Rizvi [45] have studied the two-dimensional electrostatic shock waves in epi relativistic unmagnetized plasmas consisting of relativistic thermal ions and Boltzmann distributed electrons and positrons by solving KadomtsevPetviashvili-Burgers (KPB) equation. They have shown that the ratio of ion to electron temperature, kinematic viscosity, 
positron concentration, and the relativistic ion streaming velocity significantly modify the structure of the IA shock waves. However, the effects of superthermal and subthermal electrons as well as positrons can no longer be disregarded in case of two-dimensional relativistic plasmas. It was observed in many space and laboratory plasmas that the distribution of charge particles does not follow the usual Boltzmann-Gibbs statistics which is a well-organized tool for investigating the system when the memories and microscopic interactions are short ranged. Renyi [46] and accordingly Tsallis [47] have noticed the generalization of Boltzmann-Gibbs-Shannon (BGS) entropy for statistical equilibrium having long-range interactions, long-time memories, and dissipation. The rule of composition for the two independent systems $X$ and $Y$ can be represented [48] as $S_{q}(X+Y)=S_{q}(X)+S_{q}(Y)+$ $(1-q) S_{q}(X) S_{q}(Y), q \neq 1$, where the parameters denote the degree of correlation of the system under consideration. One can be used for $S_{q} \geq 0$ and for all cases $-1<q<1$, $q=1$, and $q>1$ correspond to superthermality, isothermality, and subthermality, respectively. El-Tantawy et al. [49] have revealed that both the supersonic and subsonic electrostatic waves may exist in the nonextensive plasmas. To find the influence of charge particles nonextensitivity, the one-dimensional $q$-distribution function for $\alpha$ species can be defined [50] as $f_{\alpha}(v)=C_{q \alpha}\left\{1-(q-1)\left(m_{\alpha} v^{2} / 2 T_{\alpha}+\right.\right.$ $\left.\left.e_{\alpha} \phi / T_{\alpha}\right)\right\}^{1 /(q-1)}$, where $C_{q \alpha}$ is the normalization constant and $q$ is the strength of nonextensivity. The nonextensive density of electron and positrons can be obtained by integrating $f_{\alpha}(v)$ over the velocity space as $n_{e}=n_{e o}\left[1+(q-1) e \phi / T_{e}\right]^{(q+1) / 2(q-1)}$ and $n_{p}=n_{p o}\left[1+(q-1) e \phi / T_{p}\right]^{(q+1) / 2(q-1)}$, respectively. Therefore, the study of nonextensive relativistic plasma has received a great deal of interest from the plasma physics researchers due to its wide relevance in astrophysical and cosmological scenarios like protoneutron stars [23], stellar polytropes [24], hadronic matter and quark-gluon plasma [25], the laserplasma interaction [31], positron plasma wakefield accelerator [33], the inner region of accretion disc in the vicinity of black holes [34], and so on.

Being motivated by the above facts, this work is carried out to investigate the basic properties of IA waves in a fully ionized unmagnetized relativistic plasma system consisting of relativistic hot ions, $q$-distributed electrons, and positrons in a two-dimensional planar geometry. The plasma system is assumed to be in equilibrium with relativistic thermal ions streaming in the $x$-direction having free electrons and positrons, which is already justified by many authors $[6,7$, $11,12,44,45]$. The influences of nonextensivity parameter, positron concentration, electron-positron temperature ratio, ion-electron temperature ratio, and relativistic streaming factor on the nonlinear propagation of IA waves in unmagnetized epi plasmas are studied.

\section{Theoretical Model Equations for Two-Dimensional Plasmas}

The two-dimensional nonlinear propagation of a fully ionized unmagnetized three-component plasma system consisting of nonextensive electrons, positrons, and relativistic hot ions is considered. The charge neutrality equilibrium condition can be assumed as $n_{e 0}=n_{p 0}+n_{i 0}$, where $n_{e 0}, n_{p 0}$, and $n_{i 0}$ represent the unperturbed electrons, positrons, and ions concentrations, respectively. The concentrations of electrons and positrons are assumed to obey equilibrium $q$-distribution function. The normalized nonextensive concentrations of electron and positron can be obtained $[50,51]$ as

$$
\begin{aligned}
& n_{e}=\frac{1}{1-\alpha}[1+(q-1) \phi]^{(q+1) / 2(q-1)}, \\
& n_{p}=\frac{\alpha}{1-\alpha}[1-(q-1) \sigma \phi]^{(q+1) / 2(q-1)},
\end{aligned}
$$

where $\alpha=n_{p 0} / n_{e 0}$ and $\sigma=T_{e} / T_{p}$. It is noted that (1) and (2) are used, $-1<q<1$ for superthermal, $q>1$ for subthermal, and $q \rightarrow 1$ for isothermal electrons and positrons, respectively. Thus, the normalized basic equations governing the nonlinear dynamics of IA waves, where the phase velocity of IA waves is assumed to be smaller than the superthermal and subthermal electrons and positron velocities, can be written in the following forms:

$$
\begin{aligned}
& \frac{\partial n_{i}}{\partial t}+\frac{\partial\left(n_{i} u_{i}\right)}{\partial x}+\frac{\partial\left(n_{i} v_{i}\right)}{\partial y}=0 \\
& \frac{\partial\left(\gamma u_{i}\right)}{\partial t}+u_{i} \frac{\partial\left(\gamma u_{i}\right)}{\partial x}+v_{i} \frac{\partial\left(\gamma u_{i}\right)}{\partial y}+\frac{\partial \phi}{\partial x}+\frac{\delta}{n_{i}} \frac{\partial p_{i}}{\partial x} \\
& \quad-\mu\left(\frac{\partial^{2} u_{i}}{\partial x^{2}}+\frac{\partial^{2} u_{i}}{\partial y^{2}}\right)=0 \\
& \frac{\partial v_{i}}{\partial t}+u_{i} \frac{\partial v_{i}}{\partial x}+v_{i} \frac{\partial v_{i}}{\partial y}+\frac{\partial \phi}{\partial y}+\frac{\delta}{n_{i}} \frac{\partial p_{i}}{\partial y} \\
& \quad-\mu\left(\frac{\partial^{2} v_{i}}{\partial x^{2}}+\frac{\partial^{2} v_{i}}{\partial y^{2}}\right)=0, \\
& \frac{\partial p_{i}}{\partial t}+u_{i} \frac{\partial p_{i}}{\partial x}+v_{i} \frac{\partial p_{i}}{\partial y}+3 p_{i}\left(\frac{\partial\left(\gamma u_{i}\right)}{\partial x}+\frac{\partial v_{i}}{\partial y}\right)=0 \\
& \frac{\partial^{2} \phi}{\partial x^{2}}+\frac{\partial^{2} \phi}{\partial y^{2}}=\Omega n_{e}-(\Omega-1) n_{p}-n_{i} .
\end{aligned}
$$

Here $n_{i}$ is the ion concentration normalized by unperturbed electron concentration $\left(n_{e 0}\right), u_{i}$ and $v_{i}$, respectively, are the ion fluid velocities in the direction of $x$ and $y$ normalized by $C_{s}=\sqrt{T_{e} / m_{i}}, p_{i}$ is the pressure of ions normalized by $\left(n_{i} T_{i}\right)$, $\phi$ is the electrostatic potential normalized by $\left(T_{e} / e\right), x$ and $y$ are space variables normalized by the electron Debye radius $\lambda_{\mathrm{De}}=\sqrt{\left(T_{e} / 4 \pi n_{e 0} e^{2}\right)}$, and $t$ is the time variable normalized by the period of ion plasma $T=\sqrt{4 \pi n_{e 0} e^{2} / m_{i}}$, respectively, where $\delta=T_{i} / T_{e}, \Omega=1 /(1-\alpha)$, and $m_{i}, \phi, e, T_{e}$, and $T_{i}$ are the IA speed, ion mass, electrostatic potential, electronic charge, and electron and ion temperatures, respectively. The relativistic effect of ions is considered to be weak and can be expanded as $\gamma=1 / \sqrt{1-u_{i}^{2} / c^{2}} \approx 1+u_{i}^{2} / 2 c^{2}$. The dissipation in such collisionless plasmas occurs due to influence of ion kinematic viscosity $\left(\mu_{i}\right)$. It is well known that the ion 
kinematic viscosity for collisionless plasmas depends on the ion temperature, ion gyrofrequency, ion-ion collision time, and ion mass and widely applies in many astrophysical issues, especially in the solar wind [52]. The symbol $\mu$ is the normalized viscosity coefficient normalized by $\lambda_{\mathrm{De}} C_{s}$.

\section{Formation of KPB and KP Equations and Analytical Solutions}

To obtain a nonlinear dynamical equation in investigating the small but finite amplitude weakly relativistic IA waves in the plasma system, the stretched variables can be considered [10, 45] as

$$
\begin{aligned}
\eta & =\sqrt{\epsilon}\left(x-\lambda_{0} t\right), \\
\xi & =\epsilon y, \\
\tau & =\sqrt[3]{\epsilon} t \\
\mu & =\sqrt[2]{\epsilon} \mu_{0},
\end{aligned}
$$

$$
0<\epsilon<1 \text {, }
$$

where $\lambda_{0}$ is the linear phase velocity and $\epsilon$ is a small expansion parameter measuring the weakness of dispersion and nonlinearity. Introducing (4), (3) can be converted as follows:

$$
\begin{gathered}
\epsilon \frac{\partial n_{i}}{\partial \tau}-\lambda_{0} \frac{\partial n_{i}}{\partial \eta}+\frac{\partial}{\partial \eta}\left(n_{i} u_{i}\right)+\epsilon^{1 / 2} \frac{\partial}{\partial \xi}\left(n_{i} v_{i}\right)=0 \\
\epsilon \frac{\partial\left(\gamma u_{i}\right)}{\partial \tau}-\lambda_{0} \frac{\partial\left(\gamma u_{i}\right)}{\partial \eta}+u_{i} \frac{\partial\left(\gamma u_{i}\right)}{\partial \eta}+\epsilon^{1 / 2} v_{i} \frac{\partial\left(\gamma u_{i}\right)}{\partial \xi} \\
+\frac{\partial \phi}{\partial \eta}+\frac{\delta}{n_{i}} \frac{\partial p_{i}}{\partial \eta}-\mu_{0}\left(\epsilon \frac{\partial^{2} u_{i}}{\partial \eta^{2}}+\epsilon^{2} \frac{\partial^{2} u_{i}}{\partial \xi^{2}}\right)=0 \\
\epsilon^{3 / 2} \frac{\partial v_{i}}{\partial \tau}-\epsilon^{1 / 2} \lambda_{0} \frac{\partial v_{i}}{\partial \eta}+\epsilon^{1 / 2} u_{i} \frac{\partial v_{i}}{\partial \eta}+\epsilon v_{i} \frac{\partial v_{i}}{\partial \xi}+\epsilon \frac{\partial \phi}{\partial \xi} \\
+\epsilon \frac{\delta}{n_{i}} \frac{\partial p_{i}}{\partial \xi}-\mu_{0}\left(\epsilon^{3 / 2} \frac{\partial^{2} v_{i}}{\partial \eta^{2}}+\epsilon^{5 / 2} \frac{\partial^{2} v_{i}}{\partial \xi^{2}}\right)=0 \\
\epsilon \frac{\partial p_{i}}{\partial \tau}-\lambda_{0} \frac{\partial p_{i}}{\partial \eta}+u_{i} \frac{\partial p_{i}}{\partial \eta}+\epsilon^{1 / 2} v_{i} \frac{\partial p_{i}}{\partial \xi} \\
+3 p_{i}\left(\frac{\partial\left(\gamma u_{i}\right)}{\partial \eta}+\epsilon^{1 / 2} \frac{\partial v_{i}}{\partial \xi}\right)=0 \\
\quad-(\Omega-1)[1-(q-1) \sigma \phi]^{(q+1) / 2(q-1)}-n_{i} .
\end{gathered}
$$

Appling the reductive perturbation technique, the perturbed quantities $n_{i}, u_{i}, v_{i}, p_{i}$, and $\phi$ taking into account the charge neutrality equilibrium condition can be expanded $[45,53]$ as

$$
\begin{aligned}
& n_{i}=1+\epsilon n_{i}^{(1)}+\epsilon^{2} n_{i}^{(2)}+\cdots, \\
& p_{i}=1+\epsilon p_{i}^{(1)}+\epsilon^{2} p_{i}^{(2)}+\cdots,
\end{aligned}
$$

$$
\begin{aligned}
& u_{i}=u_{i 0}+\epsilon u_{i}^{(1)}+\epsilon^{2} u_{i}^{(2)}+\cdots, \\
& v_{i}=\epsilon^{3 / 2} v_{i}^{(1)}+\epsilon^{5 / 2} v_{i}^{(2)}+\cdots, \\
& \phi=\epsilon \phi^{(1)}+\epsilon^{2} \phi^{(2)}+\cdots .
\end{aligned}
$$

Setting (6) into (5), composed of various powers of $\epsilon$, the lowest power in $\epsilon$ gives

$$
\begin{aligned}
n_{i}^{(1)} & =\frac{1}{\gamma_{1}\left[\left(\lambda_{0}-u_{i 0}\right)^{2}-3 \delta\right]} \phi^{(1)} \\
& =\left\{\frac{(q+1)(1+\alpha \sigma)}{2(1-\alpha)}\right\} \phi^{(1)}, \\
u_{i}^{(1)} & =\frac{\left(\lambda_{0}-u_{i 0}\right)}{\gamma_{1}\left[\left(\lambda_{0}-u_{i 0}\right)^{2}-3 \delta\right]} \phi^{(1)}, \\
p_{i}^{(1)} & =\frac{3}{\left[\left(\lambda_{0}-u_{i 0}\right)^{2}-3 \delta\right]} \phi^{(1)},
\end{aligned}
$$

where $\gamma_{1}=1+1.5 \beta^{2}$, with $\beta=u_{i 0} / c$. Simplifying (7), the phase velocity is obtained as

$$
\lambda_{0}=u_{i 0}+\left\{3 \delta+\frac{2(1-\alpha)}{\gamma_{1}(q+1)(1+\alpha \sigma)}\right\}^{1 / 2} .
$$

It is seen from (8) that the phase velocity of such waves strongly depends on the related plasma parameters, but not on ion kinematic viscosity coefficient. However, collecting the next higher-order terms of $\epsilon$ yields a system of nonlinear partial differential equations (NPDEs) as

$$
\begin{gathered}
\frac{\partial n_{i}^{(1)}}{\partial \tau}-\left(\lambda_{0}-u_{i 0}\right) \frac{\partial n_{i}^{(2)}}{\partial \eta}+\frac{\partial u_{i}^{(2)}}{\partial \eta}+\frac{\partial}{\partial \eta}\left(n_{i}^{(1)} u_{i}^{(1)}\right) \\
+\frac{\partial v_{i}^{(1)}}{\partial \xi}=0, \\
\gamma_{1} \frac{\partial u_{i}^{(1)}}{\partial \tau}-\gamma_{1}\left(\lambda_{0}-u_{i 0}\right) \frac{\partial u_{i}^{(2)}}{\partial \eta} \\
+\left(\gamma_{1}-2 \gamma_{2} \frac{\lambda_{0}-u_{i 0}}{u_{i 0}}\right) u_{i}^{(1)} \frac{\partial u_{i}^{(1)}}{\partial \eta}+\frac{\partial \phi^{(2)}}{\partial \eta} \\
-\delta n_{i}^{(1)} \frac{\partial p_{i}^{(1)}}{\partial \eta}+\delta \frac{\partial p_{i}^{(2)}}{\partial \eta}-\mu_{0} \frac{\partial^{2} u_{i}^{(1)}}{\partial \eta^{2}}=0,
\end{gathered}
$$




$$
\begin{aligned}
& -\left(\lambda_{0}-u_{i 0}\right) \frac{\partial v_{i}^{(1)}}{\partial \eta}+\delta \frac{\partial p_{i}^{(1)}}{\partial \xi}+\frac{\partial \phi^{(1)}}{\partial \xi}=0 \\
& \frac{\partial p_{i}^{(1)}}{\partial \tau}-\left(\lambda_{0}-u_{i 0}\right) \frac{\partial p_{i}^{(2)}}{\partial \eta}+u_{i}^{(1)} \frac{\partial p_{i}^{(1)}}{\partial \eta}+3 \gamma_{1} \frac{\partial u_{i}^{(2)}}{\partial \eta} \\
& +3 \gamma_{1} p_{i}^{(1)} \frac{\partial u_{i}^{(1)}}{\partial \eta}+\frac{6 \gamma_{2}}{u_{i 0}} u_{i}^{(1)} \frac{\partial u_{i}^{(1)}}{\partial \eta}+3 \frac{\partial v_{i}^{(1)}}{\partial \xi}=0 \\
& \frac{\partial^{2} \phi^{(1)}}{\partial \eta^{2}}=\left\{\frac{(q+1)(1+\alpha \sigma)}{2(1-\alpha)}\right\} \phi^{(2)} \\
& -\left\{\frac{(q-3)(q+1)\left(1-\alpha \sigma^{2}\right)}{8(1-\alpha)}\right\}\left\{\phi^{(1)}\right\}^{2}-n_{i}^{(2)}
\end{aligned}
$$

where $\gamma_{2}=1.5 \beta^{2}$. Eliminating $n_{i}^{(2)}, u_{i}^{(2)}, p_{i}^{(2)}$, and $\phi^{(2)}$ from the above system of NPDEs, we obtain the following nonlinear evolution equation (NLEE):

$$
\begin{aligned}
& \frac{\partial}{\partial \eta}\left(\frac{\partial \phi^{(1)}}{\partial \tau}+A \phi^{(1)} \frac{\partial \phi^{(1)}}{\partial \eta}+B \frac{\partial^{3} \phi^{(1)}}{\partial \eta^{3}}-C \frac{\partial^{2} \phi^{(1)}}{\partial \eta^{2}}\right) \\
& \quad+D \frac{\partial^{2} \phi^{(1)}}{\partial \xi^{2}}=0 .
\end{aligned}
$$

The above equation is the two-dimensional famous KPB equation, which is very useful to investigate the nonlinear propagation of weakly relativistic IA shock structures in the plasma system considered. The nonlinearity $(A)$, dispersion $(B)$, dissipation $(C)$, and weakly transverse dispersion $(D)$ coefficients of (10) are obtained in the following forms:

$$
\begin{aligned}
A= & \left(\gamma_{1}\left(\lambda_{0}-u_{i 0}\right)-2 \gamma_{2} \frac{\left(\lambda_{0}-u_{i 0}\right)^{2}}{u_{i 0}}+\frac{6 \delta \gamma_{2}}{u_{i 0}}\right) \frac{1}{2 \mathrm{~K} \gamma_{1}^{2}} \\
& +\frac{(q-3)\left(1-\alpha \sigma^{2}\right) \mathrm{K}}{4(1+\alpha \sigma)\left(\lambda_{0}-u_{i 0}\right)}+\frac{9 \delta}{2 \mathrm{~K}\left(\lambda_{0}-u_{i 0}\right)} \\
& +\frac{1}{\gamma_{1}\left(\lambda_{0}-u_{i 0}\right)} \\
B= & \frac{\gamma_{1} \mathrm{~K}^{2}}{2\left(\lambda_{0}-u_{i 0}\right)} \\
C= & \frac{\mu_{0}}{2 \gamma_{1}} \\
D= & \frac{\gamma_{1} \mathrm{~K}+3 \delta}{2\left(\lambda_{0}-u_{i 0}\right)}
\end{aligned}
$$

where $\mathrm{K}=\left[\left(\lambda_{0}-u_{i 0}\right)^{2}-3 \delta\right]$.

To obtain the analytical solution to the NLEE of (10), the stretched variables $\eta, \xi$, and $\tau$ may be combined as $\phi^{(1)}(\eta, \xi, \tau)=\phi^{(1)}(\chi), \chi=\eta+\xi-V \tau$, where $V$ is the constant speed of the reference frame normalized by $C_{s}$ and setting the appropriate boundary conditions $\phi^{(1)} \rightarrow 0, d \phi^{(1)} / d \chi \rightarrow 0$, $d^{2} \phi^{(1)} / d \chi^{2} \rightarrow 0$, and $d^{3} \phi^{(1)} / d \chi^{3} \rightarrow 0$ as $\chi \rightarrow \pm \infty$ for localized perturbations. Equation (10) can be converted to the following form:

$$
\begin{aligned}
& \frac{d}{\partial \chi}\left(-V \frac{d \phi^{(1)}}{\partial \chi}+A \phi^{(1)} \frac{d \phi^{(1)}}{d \chi}+B \frac{d^{3} \phi^{(1)}}{d \chi^{3}}-C \frac{d^{2} \phi^{(1)}}{d \chi^{2}}\right) \\
& +D \frac{d^{2} \phi^{(1)}}{d \chi^{2}}=0 .
\end{aligned}
$$

Integrating (12) once with respect to $\chi$ and using boundary conditions for localized perturbations, one obtains

$$
\begin{aligned}
& -V \frac{d \phi^{(1)}}{\partial \chi}+A \phi^{(1)} \frac{d \phi^{(1)}}{d \chi}+B \frac{d^{3} \phi^{(1)}}{d \chi^{3}}-C \frac{d^{2} \phi^{(1)}}{d \chi^{2}} \\
& +D \frac{d \phi^{(1)}}{d \chi}=0 .
\end{aligned}
$$

Applying the Bernoulli equation method [54], the traveling wave solutions to (13) can be written as

$$
\phi^{(1)}(\chi)=a_{0}+a_{1} G(\chi)+a_{2} G^{2}(\chi)
$$

where $G(\chi)=(\Delta / 2)\{1+\tanh ((\Delta / 2) \chi)\}$ is a solution to the Bernoulli equation $d G(\chi) / d \chi=\Delta G(\chi)-G^{2}(\chi)$. The symbols $a_{0}, a_{1}, a_{2}$, and $\Delta$ are constants to be evaluated later. Substituting (14) along with the derivatives of $\phi^{(1)}(\chi)$ into (13) and collecting all the terms with the same degree of $G^{j}(\chi)(j=1,2, \ldots)$, one can obtain a system of algebraic equations for $a_{0}, a_{1}, a_{2}, \Delta$, and $V$ as follows:

$$
\begin{aligned}
& G^{1}(\xi):-V a_{1} \Delta+D a_{1} \Delta-C a_{1} \Delta^{2}+A a_{0} a_{1} \Delta \\
& \quad+B a_{1} \Delta^{3}=0, \\
& G^{2}(\xi):-2 V a_{2} \Delta-A a_{0} a_{1}+2 A a_{0} a_{2} \Delta+V a_{1} \\
& \quad-7 B a_{1} \Delta^{2}-D a_{1}+2 D a_{2} \Delta-4 C a_{2} \Delta^{2}+A a_{1}^{2} \Delta \\
& \quad+8 B a_{2} \Delta^{3}+3 C a_{1} \Delta=0, \\
& G^{3}(\xi): 3 A a_{1} a_{2} \Delta+12 B a_{1} \Delta-38 B a_{2} \Delta^{2}-2 A a_{0} a_{2} \\
& \quad+10 C a_{2} \Delta-A a_{1}^{2}-2 D a_{2}-2 C a_{1}+2 V a_{2}=0, \\
& G^{4}(\xi):-6 B a_{1}+2 A a_{2}^{2} \Delta+54 B a_{2} \Delta-3 A a_{1} a_{2} \\
& \quad-6 C a_{2}=0, \\
& G^{5}(\xi):-24 B a_{2}-2 A a_{2}^{2}=0 .
\end{aligned}
$$


Evaluating the system of algebraic equations (15), the parameters $a_{0}, a_{1}, a_{2}$, and $\Delta$ can be obtained as

$$
\begin{aligned}
\Delta & =\frac{C}{5 B}, \\
a_{0} & =\frac{25 V B+6 C^{2}-25 D B}{25 A B}, \\
a_{1} & =0, \\
a_{2} & =-\frac{12 B}{A} .
\end{aligned}
$$

Using (14) and (16), the KPB (10) has the following soliton solution:

$$
\begin{aligned}
\phi^{(1)}(\chi)= & \left\{\frac{V-D}{A}-\frac{6 C^{2}}{25 A B} \tanh \left(\frac{C}{10 B} \chi\right)\right\} \\
& +\frac{3 C^{2}}{25 A B} \operatorname{sech}^{2}\left(\frac{C}{10 B} \chi\right),
\end{aligned}
$$

where $\chi=\eta+\xi-V \tau$. It is seen that the height of the shock structure depends on the nonlinear coefficient $(A)$, dispersion coefficient $(B)$, dissipative coefficient $(C)$, and speed of the reference frame $(V)$, but the steepness of the shock structure depends only on $C$ and $B$. To identify the formation of stable shock, linearizing and integrating (13), one obtains

$$
B \frac{d^{2} \phi^{(1)}}{d \chi^{2}}-C \frac{d \phi^{(1)}}{d \chi}-(V-D) \phi^{(1)}=0
$$

The above equation is a higher-order homogeneous linear ordinary differential equation with constant coefficients. It is well known that the solutions of (18) are proportional to $e^{m \chi}$, where $m=\left(C \pm \sqrt{C^{2}-4 B(V-D)}\right) / 2 B$. It is remarkable to be noted that there will be a shock formation if $C^{2}-4 B(V-D) \geq$ 0 ; otherwise there will be oscillatory solitons. Substituting $V=6 C^{2} / 25 B+D[55]$ into the stability condition yields $C \geq 0$. The shock structures may be formed only for $C>0$. If $C=0$, (10) can be reduced as

$$
\frac{\partial}{\partial \eta}\left(\frac{\partial \phi^{(1)}}{\partial \tau}+A \phi^{(1)} \frac{\partial \phi^{(1)}}{\partial \eta}+B \frac{\partial^{3} \phi^{(1)}}{\partial \eta^{3}}\right)+D \frac{\partial^{2} \phi^{(1)}}{\partial \xi^{2}}=0
$$

This is the well-known two-dimensional KP equation, which is responsible for solitary wave or hump-shaped structures in plasmas. Therefore the solution of KPB equation can be written as

$$
\phi^{(1)}(\chi)=\phi_{0}\left\{\left[1-\tanh \left(\frac{\chi}{W_{0}}\right)\right]+\frac{1}{2} \operatorname{sech}^{2}\left(\frac{\chi}{W_{0}}\right)\right\}
$$

where $\phi_{0}=6 C^{2} / 25 A B$ is the amplitude of the shock structures and $W_{0}=10 B / C$ is the width of shock waves with $\chi=\eta+\xi-\left(6 C^{2} / 25 B+D\right) \tau$.
Again, solving the algebraic equations (15) by setting $C=$ 0 , one can determine the values of the parameters $a_{0}, a_{1}, a_{2}$, and $\Delta$ as

$$
\begin{aligned}
& \Delta=\Delta, \\
& a_{0}=\frac{V-D-B \Delta^{2}}{A}, \\
& a_{1}=\frac{12 B \Delta}{A}, \\
& a_{2}=-\frac{12 B}{A} .
\end{aligned}
$$

Using (14) and (21) and setting $\Delta=(V-D) / B$, the stationary solution to the KP equation can be obtained in the following form:

$$
\phi^{(1)}=\phi_{\mathrm{am}} \operatorname{sech}^{2}\left(\frac{\chi}{W_{1}}\right),
$$

where $\chi=\eta+\xi-V \tau, \phi_{\mathrm{am}}=3(V-D) / A$ is the amplitude of solitary waves, and $W_{1}=\sqrt{4 B /(V-D)}$ is the width of solitary waves. It should be mentioned here that there will be hump-shaped solitons if $4 B>V-D$; otherwise it will yield oscillatory waves.

\section{Results and Discussion}

The effects of unperturbed positron-electron density ratio $\left(\alpha=n_{p 0} / n_{e 0}\right)$, electron-positron temperature ratio $(\sigma=$ $\left.T_{e} / T_{p}\right)$, ion-electron temperature ratio $\left(\delta=T_{i} / T_{e}\right)$, normalized ion kinematic viscosity coefficient $\left(\mu_{0}\right)$, relativistic streaming factor $(\beta)$, and strength of electron and positrons nonextensivity $(q)$ on the nonlinear propagation of electrostatic IA waves in such relativistic plasmas have been studied numerically. The following observations are made from this investigation:

(1) The finite amplitude IA shock structures are obtained due to the involvement of dissipative term, that is, ion kinematic viscosity effect, but the finite amplitude IA solitary waves are obtained in the absence of the dissipative media. It is provided that the basic features of relativistic nonextensive plasmas support finite amplitude IA waves and strongly depend on the related plasma parameters.

(2) The influence of enhanced relativistic streaming factor (i.e., for $0 \leq \beta \leq 0.4$ ) on the nonlinear propagation of $\phi^{(1)}$ in the moving frame $\chi(\eta, \xi, \tau)$ for both cases of thermality is displayed in Figures $1(\mathrm{a})$ and $1(\mathrm{~b})$. It is observed that, with the increase of relativistic streaming factor, the magnitude of electrostatic potential of IA shock waves increases for thermal electrons and positrons, but the width of the shock waves becomes steeper in the case of subthermality.

(3) The effect of $\mu_{0}$ for superthermal and subthermal particles on the spatial electrostatic potential profiles $\phi^{(1)}$ of IA shock waves is shown in Figures 2(a) and 


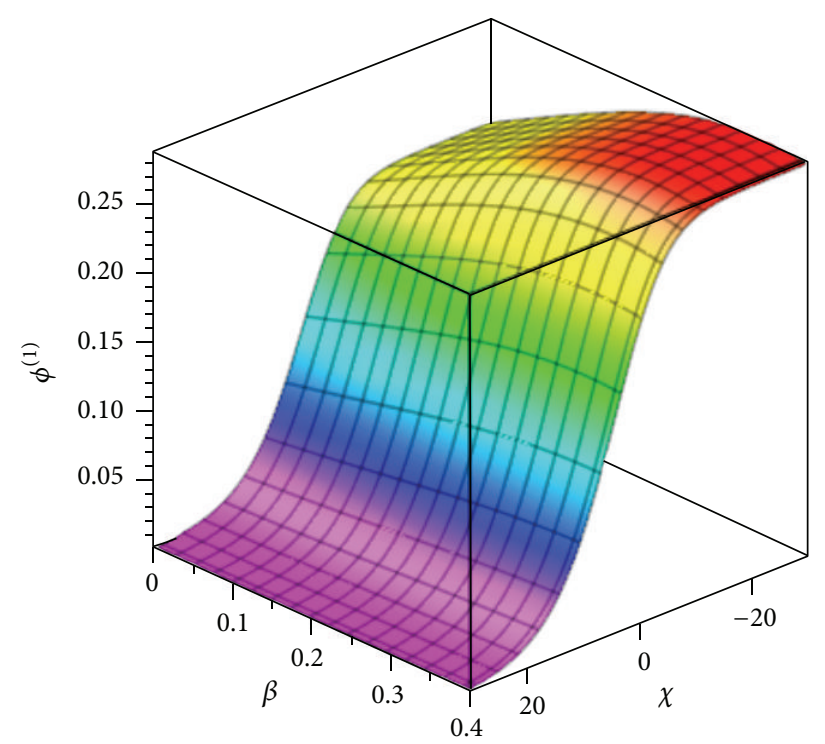

(a)

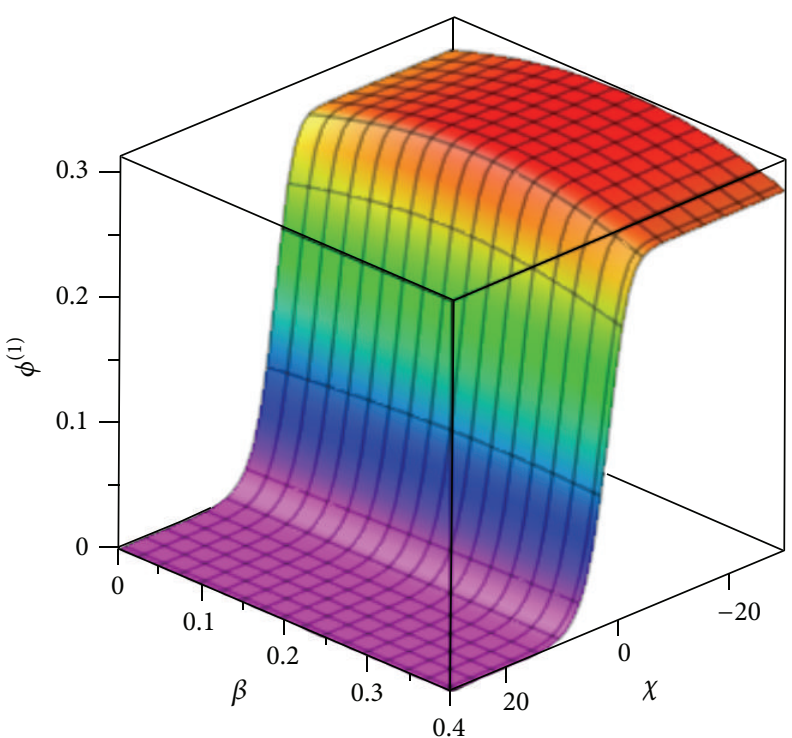

(b)

FIGURE 1: Weakly relativistic $\left(\beta=u_{i 0} / c\right)$ effects on the spatial potential $\left(\phi^{(1)}\right)$ profiles of IA shock waves in plasmas, taking $\alpha=0.1, \sigma=1$, $\delta=0.01$, and $\mu_{0}=0.96$ for the thermality parameter (a) $q=0.6$ and (b) $q=2$.

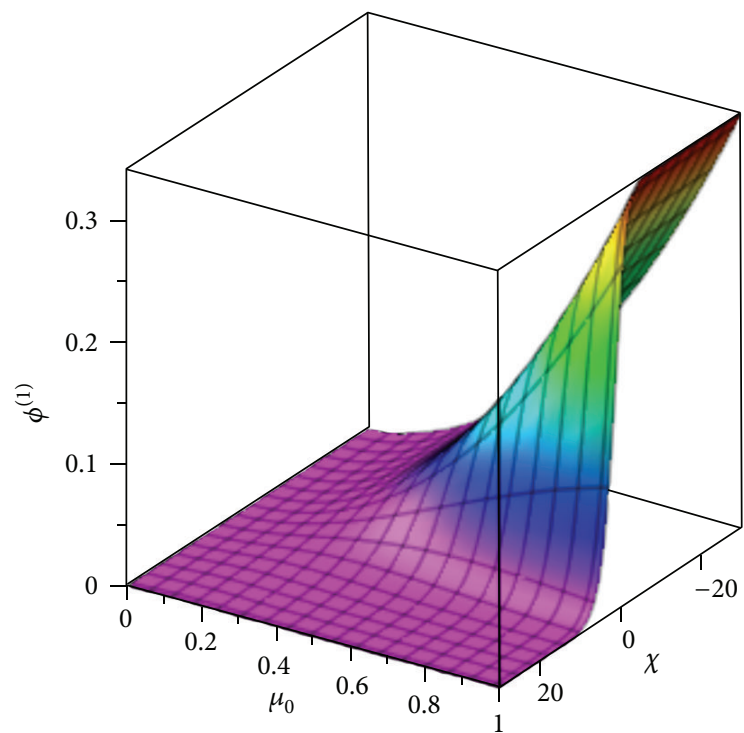

(a)

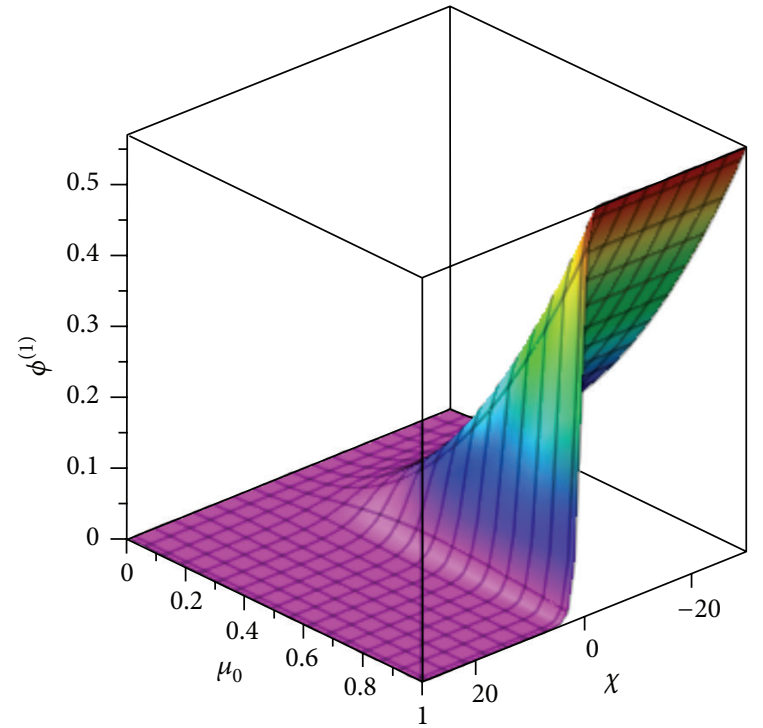

(b)

FIGURE 2: Influence of $\mu_{0}$ on the spatial potential $\left(\phi^{(1)}\right)$ profiles of IA shock waves of the plasma system considered, taking $\alpha=0.4, \sigma=1$, $\delta=0.01$, and $\beta=0.1$ for the thermality parameter (a) $q=0.6$ and (b) $q=2$.

2(b), respectively. It is seen that the amplitude of the shock waves monotonically is increased with the increase of $\mu_{0}$.

(4) The variations of spatial electrostatic potential profiles of IA waves with regard to $\chi$ and $\delta$ for both cases of thermality taking the remaining parameters constants are presented in Figures 3(a), 3(b), and 3(c). Figures 3(a) and 3(c) show that the amplitudes of the shock waves decrease for lower temperature ratio $\sigma$ of superthermal and subthermal electrons and positrons while Figure 3(b) shows that the amplitude of shock waves increases for high electron to positron temperature ratio in the case of superthermality. 


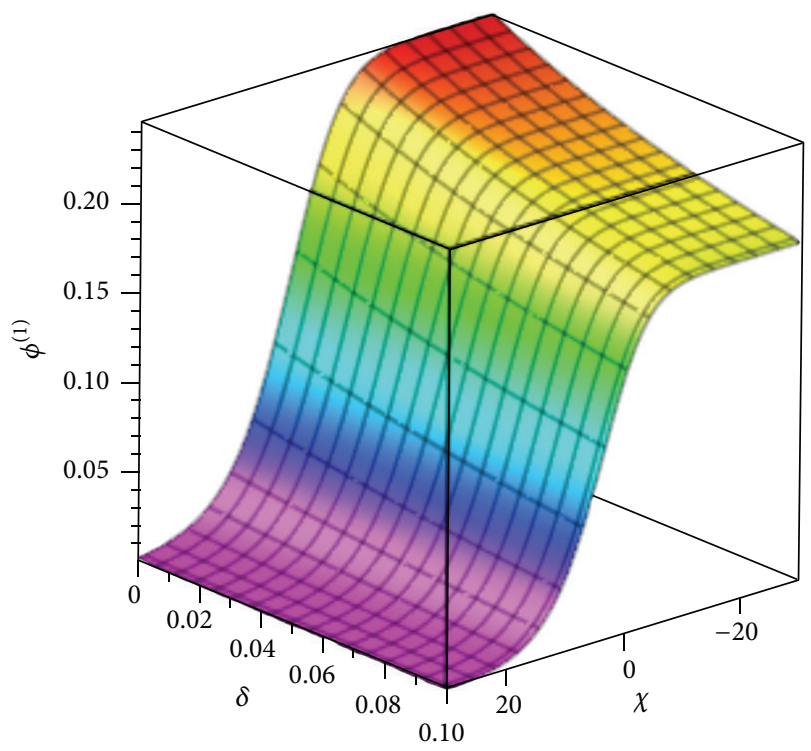

(a)

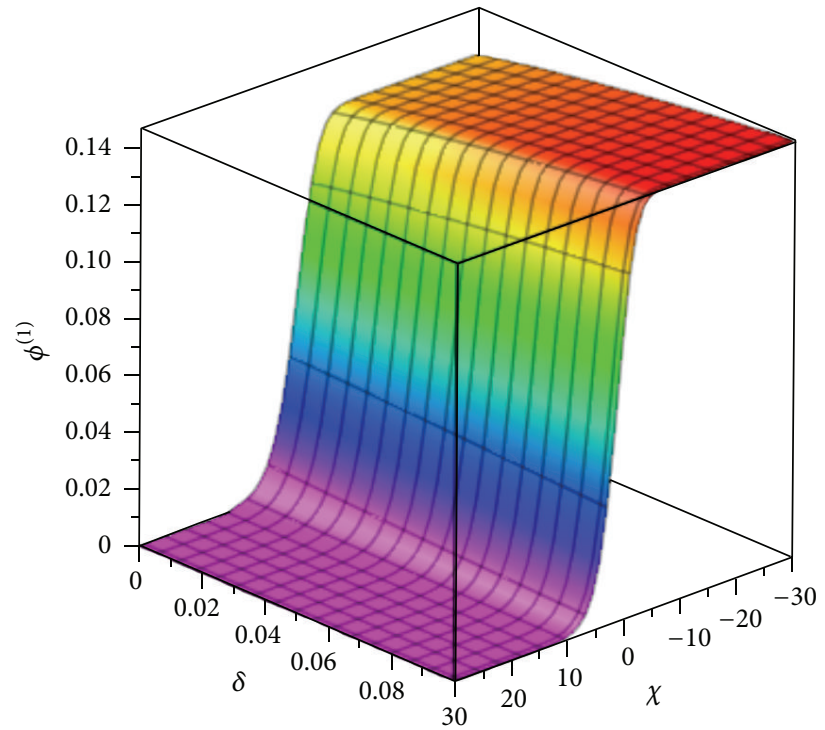

(b)

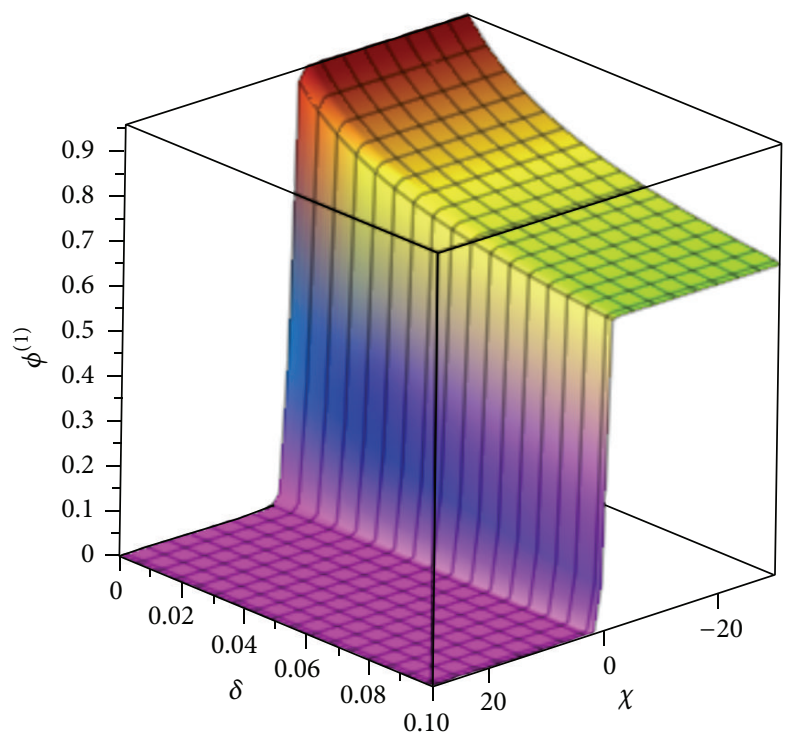

(c)

FIGURE 3: Variation of potential of electrostatic shock waves with respect to $\chi$ and $\delta$ taking the fixed values of (a) $q=0.6$ (superthermality), $\alpha=0.1, \beta=0.1, u_{i 0}=0.9, \sigma=1$ (low), and $\mu_{0}=0.95$. (b) The same values of (a) but $\sigma=10$ (high). (c) $q=4$ (subthermality), $\alpha=0.1$, $\beta=0.1, \sigma=10$, and $\mu_{0}=0.95$.

(5) The effect of $\alpha$ on spatial electrostatic potential $\phi^{(1)}$ of IA shock waves for both cases of thermality keeping the remaining parameters constants is presented in Figures 4(a), 4(b), and 4(c). The figures indicate that the shock amplitudes increase with the increase of $\alpha(0<\alpha<1)$ for superthermal as well as for subthermal electrons and positrons but sharply decrease for superthermal case. It is also found that the rarefactive shock structures are obtained for small values of $\alpha$ and negative values of $q$ with the considered values of the other parameters.
(6) Figures 5(a) and 5(b) represent the influence of $\sigma$ on the spatial electrostatic potential profiles of IA shock waves for different values of $q$ taking the constant values of the remaining parameters as mentioned in the figures, respectively. It is observed that, with the increase of $\sigma$, the amplitude of electrostatic shock waves decreases for superthermality but increases for subthermality conditions.

(7) The initial electrostatic potential profiles of IA solitary waves are displayed in Figures 6(a), 6(b), 6(c), and $6(\mathrm{~d})$ considering the different values of the 


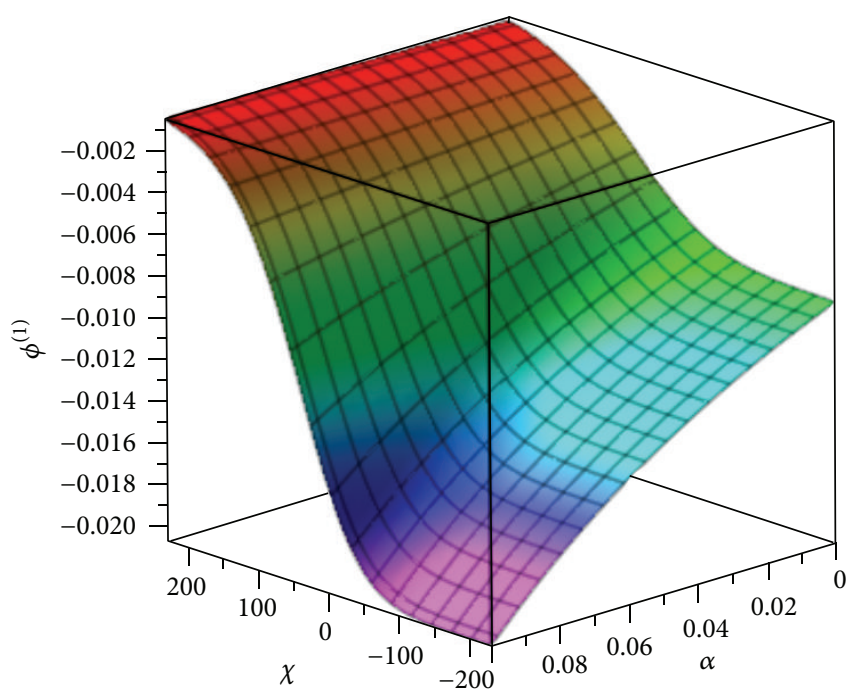

(a)

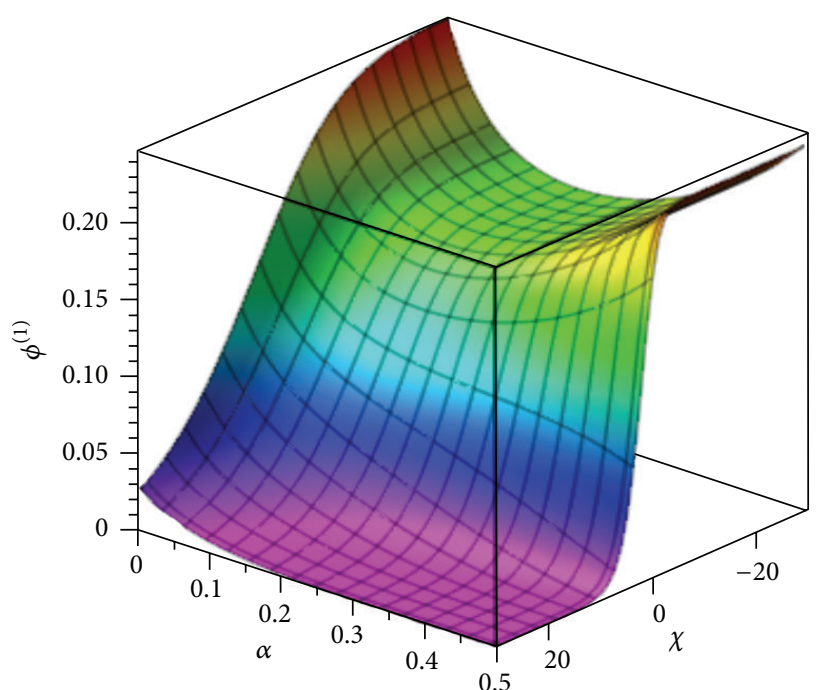

(b)

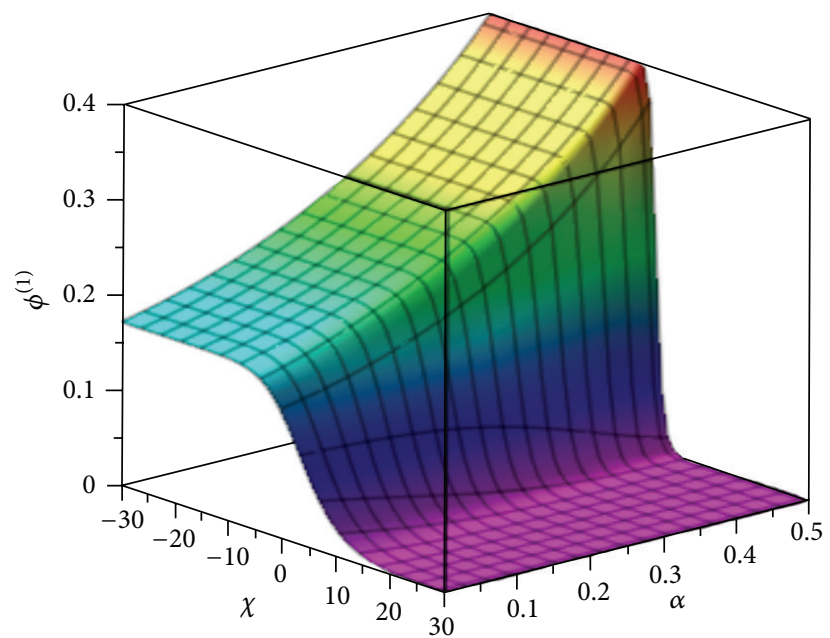

(c)

FIGURE 4: Variation of electrostatic shock waves with respect to $\chi$ and $\alpha$ for (a) $q=-0.6$ (superthermality), (b) $q=0.4$ (superthermality), and (c) $q=1.6$ (subthermality) taking the fixed values of $\alpha=0.1, \beta=0.1, \delta=0.01$, and $\mu_{0}=0.8$.

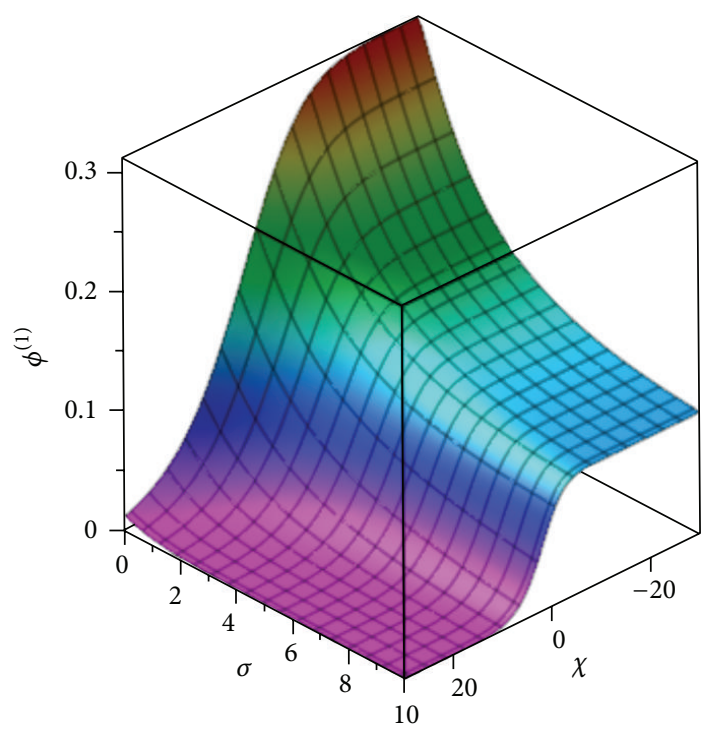

(a)

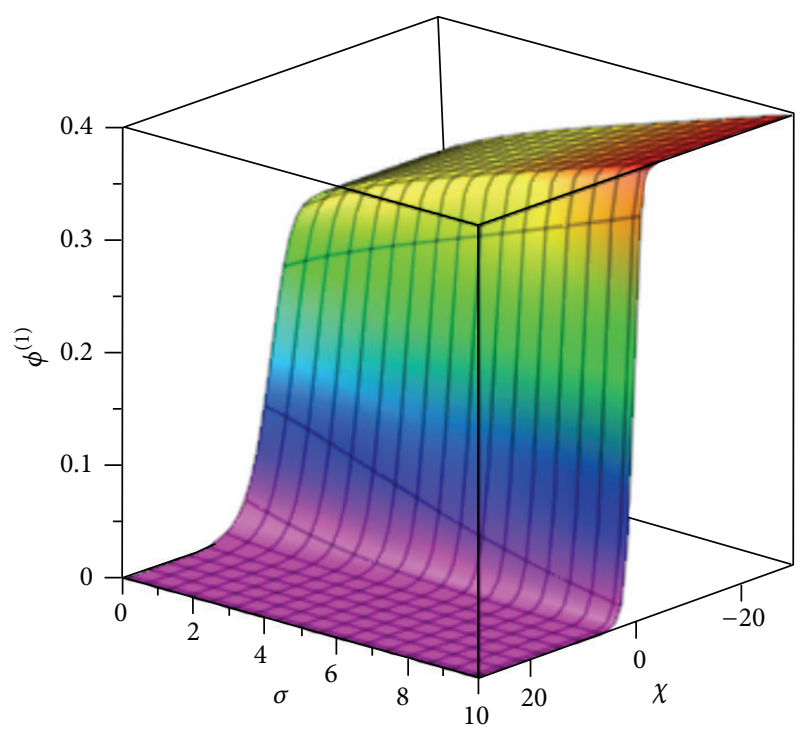

(b)

FIGURE 5: Variation of electrostatic shock waves with respect to $\chi$ and $\sigma$ for (a) $q=0.4$ (superthermality) and (b) $q=2$ (subthermality) taking the fixed values of $\alpha=0.1, \beta=0.1, \delta=0.01$, and $\mu_{0}=0.95$. 


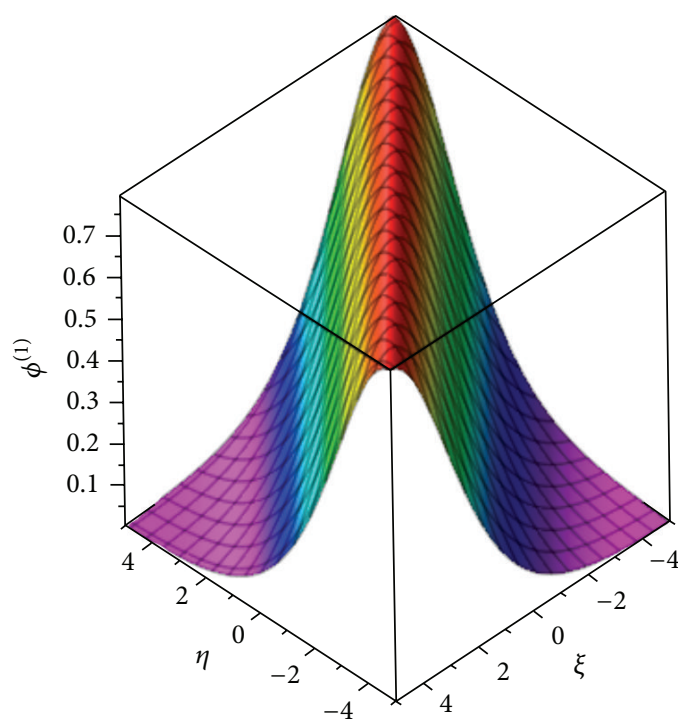

(a)

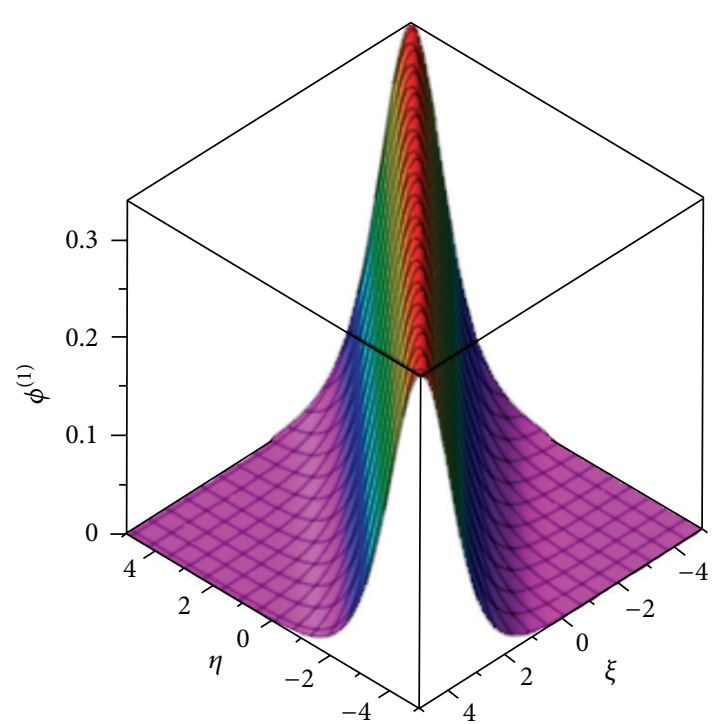

(c)

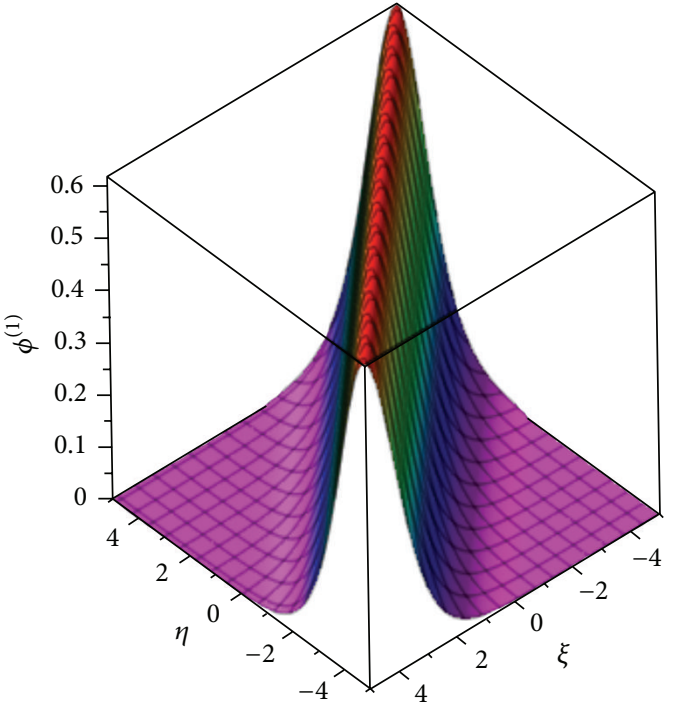

(b)

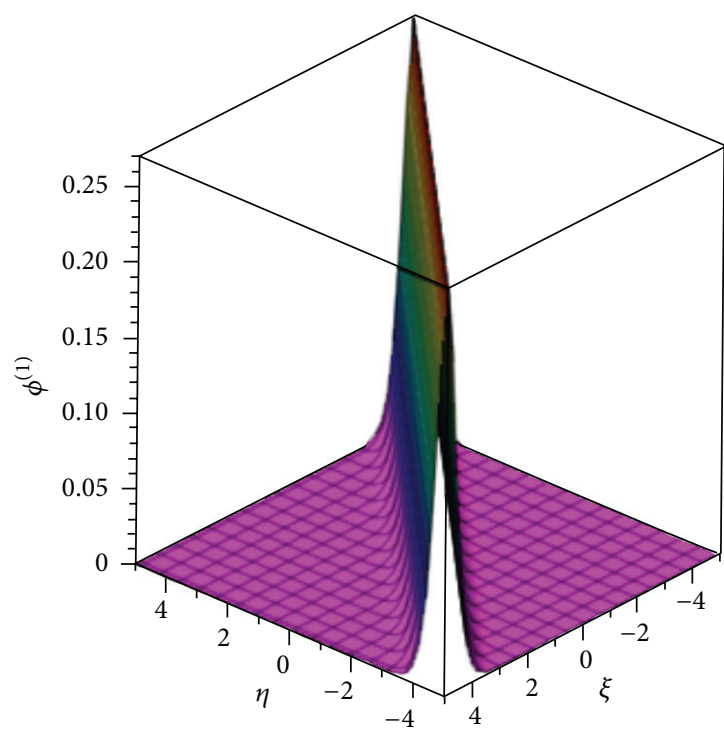

(d)

FIGURE 6: Initial potential profiles $\phi^{(1)}$ of the IA solitary waves for (a) $\alpha=0.1, q=0.6, \beta=0.1, \sigma=1, \delta=0.01$, and $V=0.75$; (b) $\alpha=0.4$ and the remaining parameters as in (a); (c) $\delta=0.1$ and the remaining parameters as in (b); and (d) $\sigma=10$ and the remaining parameters as in (b).

plasma parameters indicated in the figure caption. Visual inspection of Figures 6(a)-6(c) indicates that the amplitude as well as the width of the solitons decreases not only for superthermal case but also for subthermal electrons and positrons as $\alpha$ and $\delta$ increase for low electron to positron temperature ratio. The shape of the solitary waves becomes hump, but the amplitude becomes narrower (Figure 6(d)) for high electron to positron temperature ratio and superthermal elections and positrons.

(8) Figures $7(\mathrm{a})-7(\mathrm{c})$ present the variation of spatial electrostatic potential profiles of IA solitary waves with respect to $\beta$, whereas Figure $7(\mathrm{~d})$ presents the variation of electrostatic IA solitary waves for different values of $\tau$ considering the other parameters constants. It is observed that the amplitude of the solitary waves increases for $0<\beta<0.4$, whereas it decreases for $0.4<\beta \leq 1$. It is also observed that the IA solitary waves behave as pulse-like solitons due to the variation of time.

On the other hand, the nonlinearity, dispersion, and dissipation coefficients of KPB equation of this manuscript are equivalent to the nonlinearity, dispersion, and dissipation coefficients presented by Masood and Rizvi [45] when $q \rightarrow$ 1. Thus the results of this investigation are also in good agreement with the results provided in [45] for isothermal 


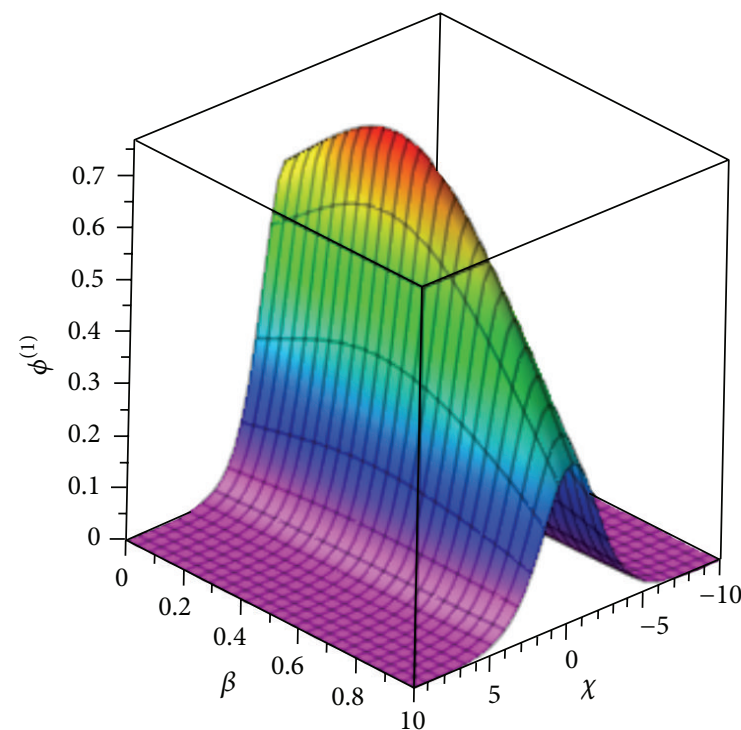

(a)

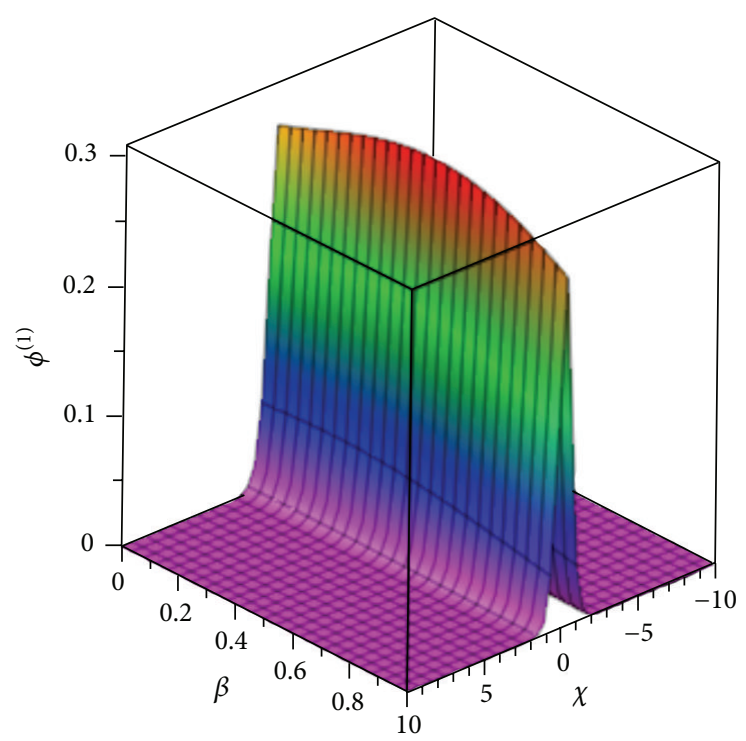

(c)

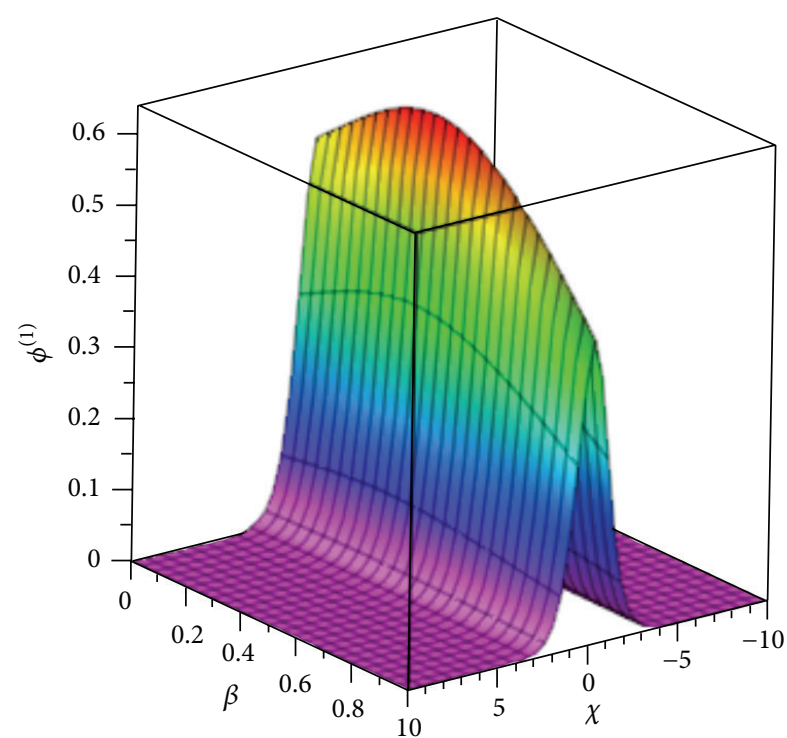

(b)

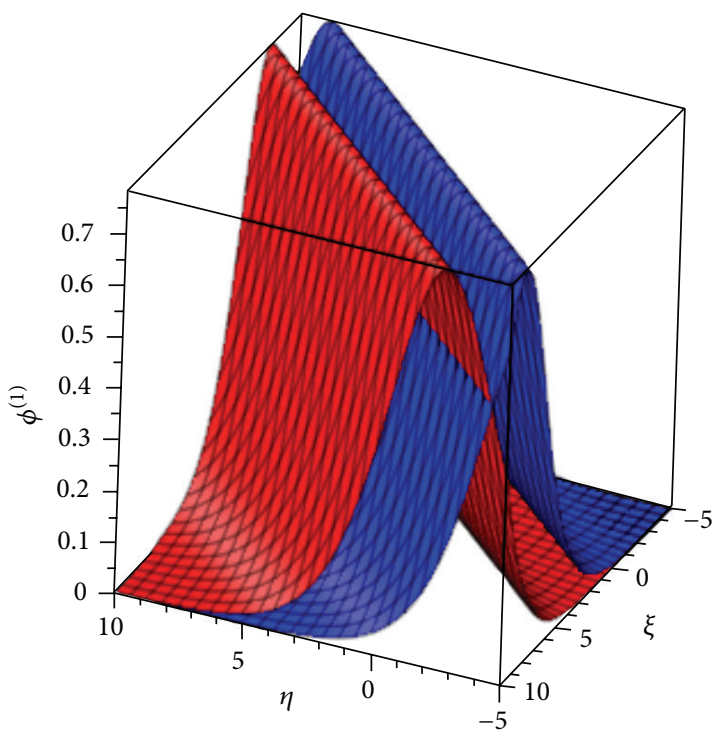

(d)

FIGURE 7: Variation of $\phi^{(1)}$ with respect to $\chi$ and $\beta$ for the different values of thermal parameter and electron to positron temperature ratio, that is, (a) $q=0.6, \sigma=1$, (b) $q=1.6, \sigma=1$, and (c) $q=0.6, \sigma=10$ taking $\alpha=0.3, \delta=0.01$, and $V=0.75$. (d) Variation of $\phi^{(1)}$ for $\tau=5$ and $\tau=10$ considering $\alpha=0.1, q=0.6, \beta=0.1, \sigma=1, \delta=0.01$, and $V=0.75$.

electrons and positrons on the nonlinear propagation of IA shock waves in the plasmas considered. Furthermore, we have also studied the nonlinear propagation of IA solitary waves using the stationary solution of the two-dimensional $\mathrm{KP}$ equation. It is seen that the compressive and rarefactive IA shock and solitary waves can be obtained for superthermal electrons and positrons, while only compressive IA waves can be found in case of subthermal electrons and positrons.

\section{Conclusion}

Two types of NLEEs are derived to study the twodimensional nonlinear electrostatic IA structures considering the unmagnetized epi plasma system consisting of relativistic ions, nonextensive electrons, and positrons that exist in many astrophysical and cosmological environments. The solutions of the equations are determined in terms of positron concentration, electron-positron and ion-electron temperature ratios, ion kinematic viscosity, strength of electron and positrons nonextensivity, and weakly relativistic streaming factor by employing the Bernoulli equation method. The fact that the phase velocity of IA waves strongly depends on the related plasma parameters, but not on ion kinematic viscosity coefficient, is investigated. The amplitudes of the IA shock waves are monotonically increasing with the increase of viscosity coefficient for both cases of thermality. The IA 
solitary waves become hump- and pulse-shaped due to relativistic effect in the absence of the dissipative media, that is, viscosity coefficient. The compressive and rarefactive IA shock and solitary waves are obtained for superthermal electrons and positrons, while only compressive IA waves are found in case of subthermal electrons and positrons. Finally, this work may be helpful for understanding the structures of IA waves for laboratory and astrophysical plasmas. This work is done for nonlinear shock and solitary propagation in our plasma model in case of planar geometry which is only valid for small but finite amplitude ion acoustic structures. But still there are so many possibilities to make further investigation on nonlinear analysis of shock waves, solitary waves, vortices, solitons, double layers, and so forth, with nonextensive electrons, positrons, and relativistic thermal ions using this same plasma model. These are also the problems of great importance in plasma physics for better understanding the characteristics of astrophysical compact objects but beyond the scope of the present work.

\section{Competing Interests}

The authors declare that they have no competing interests.

\section{References}

[1] G. C. Das and S. N. Paul, "Ion-acoustic solitary waves in relativistic plasmas," Physics of Fluids, vol. 28, no. 3, pp. 823-825, 1985.

[2] Y. Nejoh, "The effect of the ion temperature on the ion acoustic solitary waves in a collisionless relativistic plasma," Journal of Plasma Physics, vol. 37, no. 3, pp. 487-495, 1987.

[3] Y. Wang, Z. Zhou, X. Jiang et al., "Cylindrical KadomtsevPetviashvili equation for relativistically magnetosonic solitary wave in the collisionless plasma," Physics Letters. A, vol. 355, no. 4-5, pp. 386-389, 2006.

[4] W. M. Moslem, I. Kourakis, P. K. Shukla, and R. Schlickeiser, "Nonlinear excitations in electron-positron-ion plasmas in accretion disks of active galactic nuclei," Physics of Plasmas, vol. 14, no. 10, Article ID 102901, 2007.

[5] W. Masood, N. Jehan, A. M. Mirza, and P. H. Sakanaka, "Planar and non-planar ion acoustic shock waves in electron-positronion plasmas," Physics Letters A, vol. 372, no. 23, pp. 4279-4282, 2008.

[6] T. S. Gill, A. Singh, H. Kaur, N. S. Saini, and P. Bala, "Ionacoustic solitons in weakly relativistic plasma containing electron-positron and ion," Physics Letters, Section A: General, Atomic and Solid State Physics, vol. 361, no. 4-5, pp. 364-367, 2007.

[7] T. S. Gill, A. S. Bains, and N. S. Saini, "Ion acoustic soliton in weakly relativistic magnetized electron-positron- ion plasma," Canadian Journal of Physics, vol. 87, no. 8, pp. 861-866, 2009.

[8] M. Tribeche and L. Djebarni, "Electron-acoustic solitary waves in a nonextensive plasma," Physics of Plasmas, vol. 17, no. 12, Article ID 124502, 2010.

[9] H. R. Pakzad, "Ion acoustic shock waves in dissipative plasma with superthermal electrons and positrons," Astrophysics and Space Science, vol. 331, no. 1, pp. 169-174, 2011.

[10] H. R. Pakzad and M. Tribeche, "Nonlinear propagation of ion-acoustic shock waves in dissipative electron-positron-ion plasmas with superthermal electrons and relativistic ions," Journal of Fusion Energy, vol. 32, no. 2, pp. 171-176, 2013.

[11] M. G. Hafez and M. R. Talukder, "Ion acoustic solitary waves in plasmas with nonextensive electrons, Boltzmann positrons and relativistic thermal ions," Astrophysics and Space Science, vol. 359, article 27, 2015.

[12] M. G. Hafez, M. R. Talukder, and R. Sakthivel, "Ion acoustic solitary waves in plasmas with nonextensive distributed electrons, positrons and relativistic thermal ions," Indian Journal of Physics, vol. 90, no. 5, pp. 603-611, 2016.

[13] K. Javidan and D. Saadatmand, "Effect of high relativistic ions on ion acoustic solitons in electron-ion-positron plasmas with nonthermal electrons and thermal positrons," Astrophysics and Space Science, vol. 333, no. 2, pp. 471-475, 2011.

[14] H. K. Malik, "Effect of electron inertia on KP solitons in a relativistic plasma," Physica D: Nonlinear Phenomena, vol. 125, no. 3-4, pp. 295-301, 1999.

[15] H. K. Malik, "Ion acoustic solitons in a weakly relativistic magnetized warm plasma," Physical Review E, vol. 54, no. 5, pp. 5844-5847, 1996.

[16] H. K. Malik, "Ion acoustic solitons in a relativistic warm plasma with density gradient," IEEE Transactions on Plasma Science, vol. 23, no. 5, pp. 813-815, 1995.

[17] R. Malik, H. K. Malik, and S. C. Kaushik, "Study of solitary waves in e-p pair plasma: effect of weakly relativistic species," in Proceedings of the International Conference on Recent Trends in Applied Physics and Material Science, RAM 2013, AIP Conference Proceedings, pp. 1111-1112, Bikaner, India, February 2013.

[18] K. Singh, V. Kumar, and H. K. Malik, "Electron inertia contribution to soliton evolution in an inhomogeneous weakly relativistic two-fluid plasma," Physics of Plasmas, vol. 12, no. 7, Article ID 072302, 2005.

[19] K. Singh, V. Kumar, and H. K. Malik, "Electron inertia effect on small amplitude solitons in a weakly relativistic two-fluid plasma," Physics of Plasmas, vol. 12, no. 5, Article ID 052103, 2005.

[20] D. K. Singh and H. K. Malik, "Solitons in inhomogeneous magnetized negative ion containing plasma with two temperature nonisothermal electrons," IEEE Transactions on Plasma Science, vol. 36, no. 2, pp. 462-468, 2008.

[21] R. Malik, H. K. Malik, and S. C. Kaushik, "Soliton propagation in a moving electron-positron pair plasma having negatively charged dust grains," Physics of Plasmas, vol. 19, no. 3, Article ID 032107, 2012.

[22] H. K. Malik and R. Malik, "Unperturbed state and solitary structures in an electron-positron plasma having dust impurity and density inhomogeneity," Journal of Plasma Physics, vol. 80, no. 4, pp. 629-641, 2014.

[23] A. Lavagno and D. Pigato, "Nonextensive statistical effects in protoneutron stars," European Physical Journal A, vol. 47, article 52, 2011.

[24] A. R. Plastino and A. Plastino, "Stellar polytropes and Tsallis' entropy," Physics Letters. A, vol. 174, no. 5-6, pp. 384-386, 1993.

[25] G. Gervino, A. Lavagno, and D. Pigato, "Nonextensive statistical effects in the quark-gluon plasma formation at relativistic heavy-ion collisions energies," Central European Journal of Physics, vol. 10, no. 3, pp. 594-601, 2012.

[26] H. R. Miller and P. J. Wiita, Active Galactic Nuclei, Springer, Berlin, Germany, 1987.

[27] F. C. Michel, “Theory of pulsar magnetospheres," Reviews of Modern Physics, vol. 54, no. 1, pp. 1-66, 1982. 
[28] P. Goldreich and W. H. Julian, "Pulsar electrodynamics," The Astrophysical Journal, vol. 157, p. 869, 1969.

[29] F. C. Michel, Theory of Neutron Star Magnetoshpere, Chicago University Press, Chicago, Ill, USA, 1991.

[30] M. L. Burns, Positron-Electron Pairs in Astrophysics, American Institute of Physics, New York, NY, USA, 1983.

[31] B. Shen and J. Meyer-ter-Vehn, "Pair and $\gamma$-photon production from a thin foil confined by two laser pulses," Physical Review E, vol. 65, no. 1, Article ID 016405, 6 pages, 2002.

[32] E. P. Liang, S. C. Wilks, and M. Tabak, "Pair production by ultraintense lasers," Physical Review Letters, vol. 81, no. 22, pp. 4887-4890, 1998.

[33] X. Wang, R. Ischebeck, P. Muggli et al., "Positron injection and acceleration on the wake driven by an electron beam in a foiland-gas plasma," Physical Review Letters, vol. 101, no. 12, Article ID 124801, 2008.

[34] F. D. Steffen and M. H. Thoma, "Hard thermal photon production in relativistic heavy ion collisions," Physics Letters B, vol. 510, no. 1-4, pp. 98-106, 2001.

[35] J. Arons, "Some problems of pulsar physics or I'm madly in love with electricity," Space Science Reviews, vol. 24, no. 4, pp. 437$510,1979$.

[36] A. Spitkovsky, "On the structure of relativistic collisionless shocks in electron-ion plasmas," The Astrophysical Journal, vol. 673, no. 1, pp. L39-L42, 2008.

[37] C. Grabbe, "Wave propagation effects of broadband electrostatic noise in the magnetotail," Journal of Geophysical Research, vol. 94, no. A12, pp. 17299-17304, 1989.

[38] H. Chen, A. Link, Y. Sentoku et al., "The scaling of electron and positron generation in intense laser-solid interactions," Physics of Plasmas, vol. 22, no. 5, Article ID 056705, 2015.

[39] R. Bostrom, "Observations of weak double layers on auroral field lines," IEEE Transactions on Plasma Science, vol. 20, no. 6, pp. 756-763, 1992.

[40] W. Theobald, R. Nora, W. Seka et al., "Spherical strongshock generation for shock-ignition inertial fusion," Physics of Plasmas, vol. 22, Article ID 056310, 2015.

[41] M. Q. Tran, "Ion acoustic solitons in a plasma: a review of their experimental properties and related theories," Physica Scripta, vol. 20, no. 3-4, pp. 317-327, 1979.

[42] Y. Nakamura, "Experiments on ion-acoustic solitons in plasmas invited review article," IEEE Transactions on Plasma Science, vol. 10, no. 3, pp. 180-195, 1982.

[43] K. E. Lonngren, "Soliton experiments in plasmas," Plasma Physics, vol. 25, no. 9, p. 943, 1983.

[44] J. Han, S. Du, and W. Duan, "Ion-acoustic solitary waves and their interaction in a weakly relativistic two-dimensional thermal plasma," Physics of Plasmas, vol. 15, no. 11, Article ID 112104, 2008.

[45] W. Masood and H. Rizvi, "Two dimensional electrostatic shock waves in relativistic electron positron ion plasmas," Physics of Plasmas, vol. 17, no. 5, Article ID 052314, 2010.

[46] A. Renyi, "On a new axiomatic theory of probability," Acta Mathematica Academiae Scientiarum Hungaricae, vol. 6, pp. 285-335, 1955.

[47] C. Tsallis, "Possible generalization of Boltzmann-Gibbs statistics," Journal of Statistical Physics, vol. 52, no. 1-2, pp. 479-487, 1988.

[48] C. Tsallis, "Some comments on Boltzmann-Gibbs statistical mechanics," Chaos, Solitons and Fractals, vol. 6, pp. 539-559, 1995.
[49] S. A. El-Tantawy, M. Tribeche, and W. M. Moslem, "Nonlinear structures in a nonextensive electron-positron-ion magnetoplasma," Physics of Plasmas, vol. 19, no. 3, Article ID 032104, 2012.

[50] M. G. Hafez, M. R. Talukder, and M. H. Ali, "Ion acoustic shock and solitary waves in highly relativistic plasmas with nonextensive electrons and positrons," Physics of Plasmas, vol. 23, no. 1, Article ID 012902, 2016.

[51] P. Eslami, M. Mottaghizadeh, and H. R. Pakzad, "Modulational instability of ion acoustic waves in e-p-i plasmas with electrons and positrons following a q-nonextensive distribution," Physics of Plasmas, vol. 18, no. 10, article 102313, 2011.

[52] M. K. Verma, "Nonclassical viscosity and resistivity of the solar wind plasma," Journal of Geophysical Research: Space Physics A, vol. 101, no. 12, pp. 27543-27548, 1996.

[53] A. N. Dev, M. K. Deka, R. Subedi, and J. Sarma, "Dust acoustic compressive waves in a warm dusty plasma having nonthermal ions and non-isothermal electrons," Plasma Science and Technology, vol. 17, no. 9, pp. 721-727, 2015.

[54] M. Mirzazadeh, M. Eslami, E. Zerrad, M. F. Mahmood, A. Biswas, and M. Belic, "Optical solitons in nonlinear directional couplers by sine-cosine function method and Bernoulli's equation approach," Nonlinear Dynamics, vol. 81, no. 4, pp. 1933-1949, 2015.

[55] M. G. Hafez, M. R. Talukder, and M. H. Ali, "Comment on 'Electrostatic compressive and rarefactive shocks and solitons in relativistic plasmas occurring in polar regions of pulsar," Astrophysics and Space Science, vol. 361, article 154, 2016. 


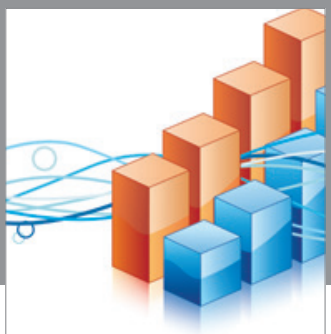

Advances in

Operations Research

vatem alat4

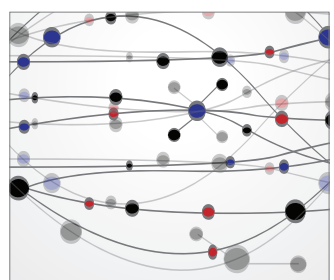

\section{The Scientific} World Journal
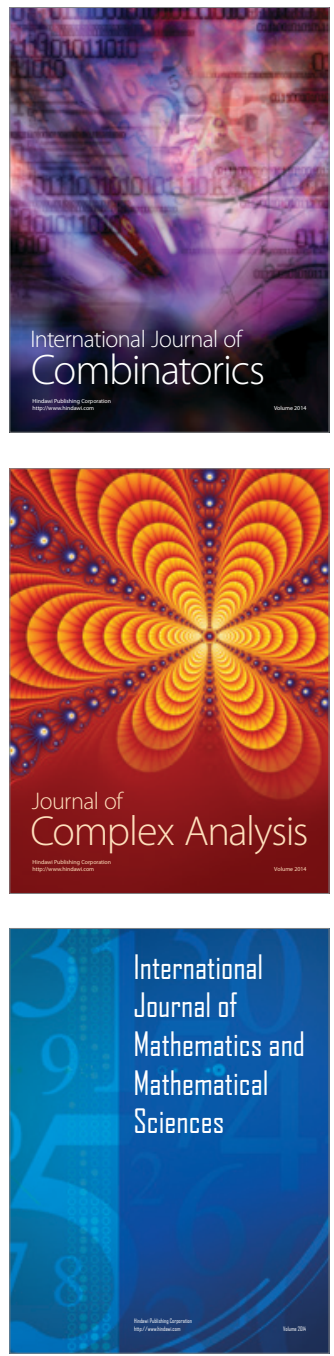
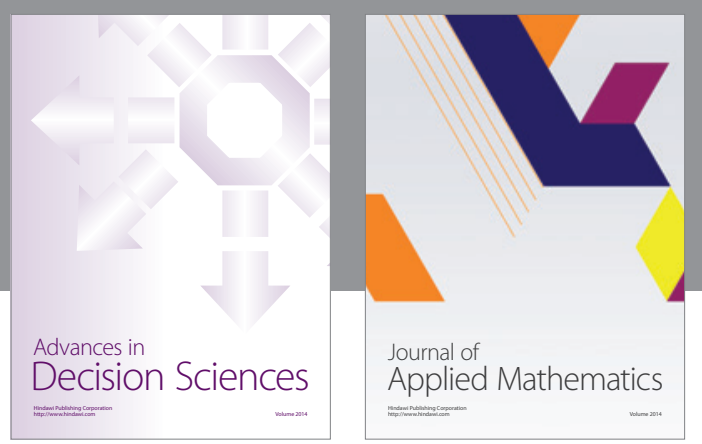

Algebra

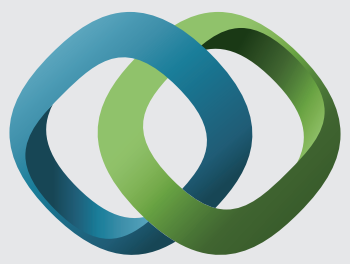

\section{Hindawi}

Submit your manuscripts at

http://www.hindawi.com
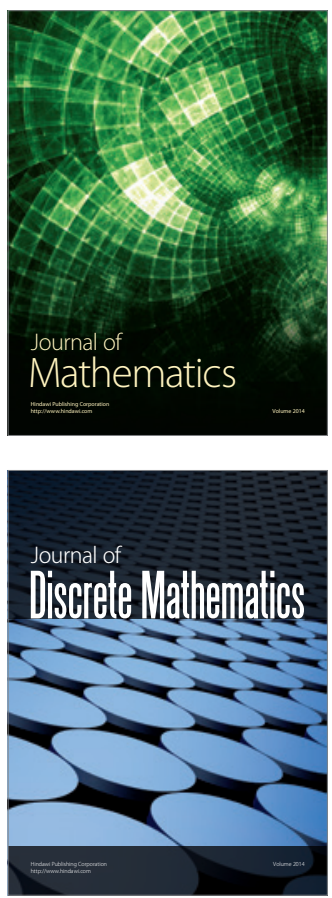

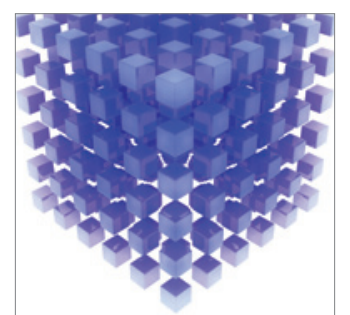

Mathematical Problems in Engineering
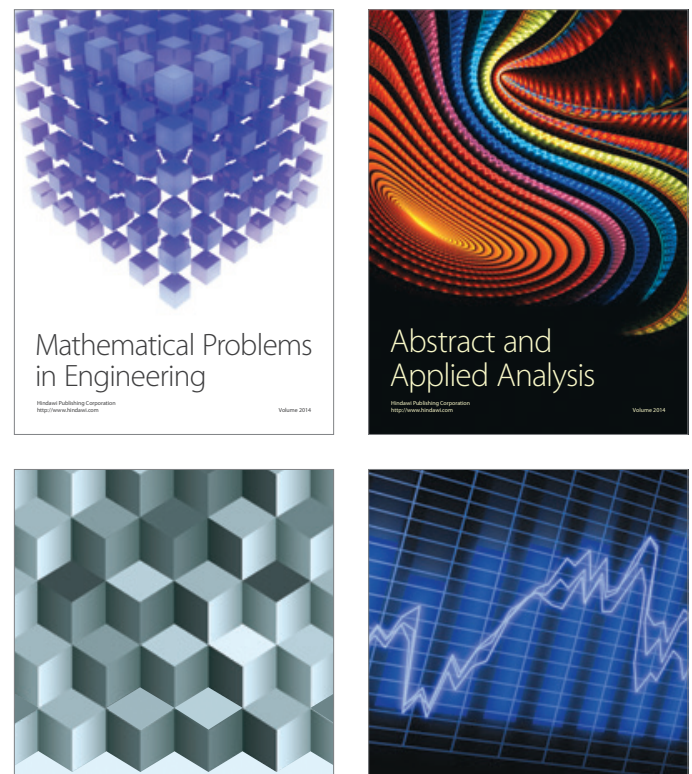

Journal of

Function Spaces

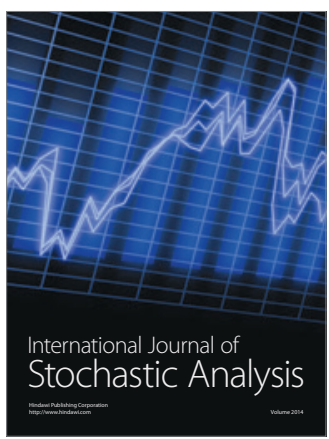

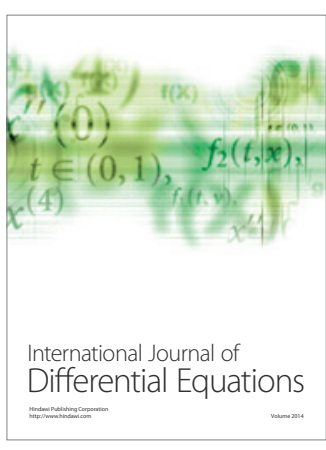
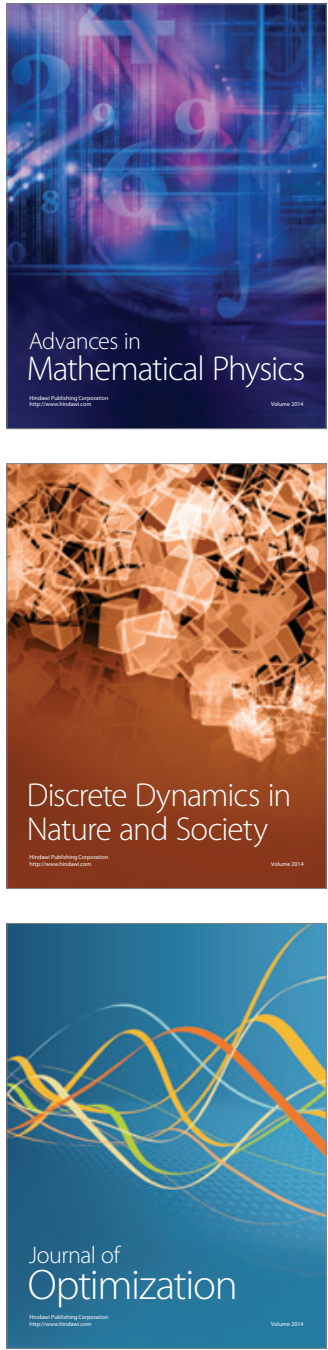